Abstract: We show the both amplitude and spectrum dynamics of few-cycle laser pulses in two-photon Raman-type self-induced transparency in $A r^{+}$ions, that permit to generate half-cycle laser pulses (FWHM) with more high amplitudes and photon energy as well as spectral widths.

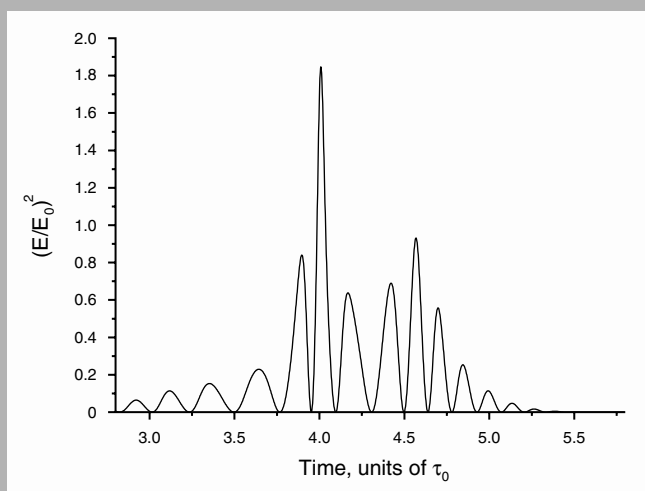

\title{
Two-photon Raman-type self-induced transparency for few-cycle laser pulses
}

\author{
I.P. Prokopovich* \\ Institute of Nuclear Problems, Belarusian State University, 11 Bobruiskaya Str., 220050 Minsk, Belarus \\ Received: 2 October 2004, Accepted: 12 October 2004 \\ Published online: 11 November 2004
}

Key words: two-photon self-induced transparency; Raman; half-cycle laser pulses; spectral supercontinuum

PACS: 42.50.Gy, 42.50.Md, 42.65.Dr, 42.65.Re

\section{Introduction}

Self-induced transparency (SIT) due to a one-photon resonant transition (one-photon SIT) has been predicted for the first time by MaCall and Hahn [1], verified experimentally both by themselves and later by other authors (see, for example, [1-4] and references cited therein). Coherent two-photon propagation exhibiting SIT (two-photon SIT), in which twice the propagation frequency $\omega$ is resonant with a two-level system

$\omega_{21}=\omega_{1}+\omega_{2}, \quad \omega_{2}=\omega_{1}=\omega$,

the $\omega_{21}$ is frequency of transition, has been predicted and analyzed by Belenov and Poluektov [5]. Tan-no et al. observed [6] the two-photon SIT in potassium $\left(4 \mathrm{~S}_{1 / 2}-6 S_{1 / 2}\right.$ transition and $\left.\omega_{2} \neq \omega_{1}\right)$ for the first time. Gridin et al. observed the two-photon SIT in $\mathrm{Nd}^{3+}\left({ }^{4} \mathrm{I}_{9 / 2}-{ }^{4} \mathrm{G}_{7 / 2}\right.$ transition and $\omega_{2}=\omega_{1}$ ) [7]. The transient pulse behavior has been studied both for direct two-photon resonant transitions

$\omega_{21}=\omega_{1}+\omega_{2}$,

and Raman-type transitions

$\omega_{21}=\omega_{1}-\omega_{2}$,

in many work (see, for example [8-11]). In all these works the approximation of a slowly varying amplitude (SWA) was used. However, the SWA is not valid for few-cycle laser pulses that is available at present (see, for example [12]). First the studies the one-photon SIT without using SWA was performed by Bullough, Eilbeck, et al. [13-15]. If a dimensionless constant

$\alpha_{s}^{(1)}=\frac{4 \pi N d_{21}}{\hbar \omega_{21}}$

(where the $N$ is an atomic density, the $d_{21}$ is a matrix element of the one-photon resonant transition with frequency $\left.\omega_{21}\right)$ is less than 1 then the reduced Maxvell-Bloch

* Corresponding author: e-mail: prok@inp.minsk.by 
equations (the Maxvell-Bloch equations in which only backscattering is neglected) provides good description SIT for pulses with duration up to half-cycle of the electromagnetic field. For example, for a typical value of $d_{21}, 10^{-18}$ cgs units, this is a good approximation (to within 1\%) at $N=10^{18} \mathrm{~cm}^{-3}$.

In our work [16] a two-photon Raman-type SIT (RSIT) for few-cycle laser pulses had been predicted and an equation for the change in the total energy had been derived [16]. However, the transient pulse behavior was not studied. On the other hand, it is known that a two-photon interaction have many crucial different properties [5-11], as regarding a one-photon interaction, that must influence on transient pulse behavior for few-cycle field. These properties are an instantaneous intensity-dependent cross section, continuous frequency-modulating and pulse reshaping. It has been shown that the $2 \pi$ pulse RSIT in conditions of stimulated Raman self-scattering (SRSS)permit to generate a spectral supercontinuum [17-20]. First a breakup of a few-cycle $4 \pi$ pulse into two $2 \pi$ pulse was observed in our simulation on SRSS [21] with pulses containing three and four optical cycles (full width at half maximum (FWHM)).

Here we report new results on transient pulse behavior at RSIT. We investigate the field and spectral dynamics of $2 n-\pi$ few-cycle laser pulses. We show that, breakup of few-cycle $4 \pi$ and $6 \pi$ pulses into $2 \pi$-pulses at RSIT occur. Such breakup with conserved total energy of the initial pulse are unique signatures of SIT. However, $2 \pi$-pulse at RSIT is nonstationary one on its spectrum and shape. This breakup and two-photon repumping an energy from front of the $2 \pi$-pulse into its backside permit to generate single and double half-cycle pulses (FWHM) having more high amplitudes and frequency as compared with initial pulse. The spectrum of the pulse is supercontinuum that is broadened continuously with increasing propagation length.

\section{Basic equations}

We will consider the propagation of a laser pulse with duration $\tau$ which satisfies the condition SRSS [16-22]

$\delta \equiv \frac{\tau \omega_{21}}{2 \pi}<1$,

where $\hbar \omega_{21}=\epsilon_{2}-\epsilon_{1}$ is the difference in level energies participating in the regime (1) of the SRSS in the medium. We will consider the stimulated Raman scattering within the framework of a semiclassical model, when dynamics of the laser pulse field $E$ is described by the wave equation

$\frac{\partial^{2} E}{\partial z^{2}}-\frac{1}{v^{2}} \frac{\partial^{2} E}{\partial t^{2}}=\frac{4 \pi}{c^{2}} \frac{\partial^{2} P_{n l}}{\partial t^{2}}$,

where $v$ is the speed of the laser pulse arising from the linear contribution to polarization, and $P_{n l}$ is the nonlinear contribution to polarization, caused by stimulated Raman process. The Raman-active medium is described by a two-level model where the direct one-photon transitions between the levels is forbidden in electro-dipole approximation, but permitted for two-photon process like the SRSS. The frequency of Raman transition $\omega_{21}$ is considered to be much less than the central frequency $\omega_{c}$ of the initial laser pulse field $E$. For example, such situation can take place for the fine structure ${ }^{2} P_{3 / 2}^{0}-{ }^{2} P_{1 / 2}^{0}$ levels of $\mathrm{Ar}^{+}$ions (where ground state ${ }^{2} P_{3 / 2}^{0}$ and ${ }^{2} P_{1 / 2}^{0}$ forms a two-level system with the frequency transition $\omega_{21} / 2 \pi c=1432 \mathrm{~cm}^{-1}$, and all other levels are removed on $\Delta>108723 \mathrm{~cm}^{-1}$ [23]) and 16-fs (or shorter) pulses (that have the spectral width $\Delta \omega_{\tau}=2084 \mathrm{~cm}^{-1}$ (or more) with central wavelength $800 \mathrm{~nm}$ ). The energy two-photon interaction of broad spectrum pulse

$V=-k \iint \alpha\left(\omega_{2}, \omega_{1}\right) E\left(\omega_{2}\right) E\left(\omega_{1}\right) d \omega_{2} d \omega_{1}$,

where normalization factor $k=\left(\Delta \omega_{\tau}\right)^{-2} / 2$, and $\alpha$ is the operator of dipole polarizability of the medium. In our case the condition of strong two-photon resonance $\left(\omega_{1}-\omega_{2}=\omega_{21}\right)$ is completely satisfied by Eq. (1). (So perturbation theory can not be used.) At ordinary temperature and atomic density $N=3 \times 10^{18} \mathrm{~cm}^{-3}$ the Doppler dephasing time $T_{D}^{*}$, the polarization relaxation time $T_{2}$, and the time of population relaxation $T_{1}$ are estimated from experimental data to be ps-range $\left(T_{D}^{*} \simeq 100 \mathrm{ps}, T_{2}>2 \mathrm{ps}\right.$, $T_{2} \ll T_{1}$ ) [24,25]. Hence, first, the condition $\tau<T_{2}$ of coherent interaction between the pulse and the two-photon transition is fulfilled very good. Second, the inhomogeneous broadening of the resonance transition can be neglected $\left(\tau / T_{D}^{*}<10^{-3}\right)$. Third, since the dipole polarizability in rare gases at two-photon resonance is increased by about 3 orders of magnitude (experimental results show that susceptibility $\chi$ in rare gases at two-photon resonance can be increased by up to 7 orders of magnitude [26] ( $\chi \sim$ $\left|\alpha_{12}\right|^{2}$, where $\alpha_{12}$ is a matrix element of the polarizability corresponding to the Raman transition with frequency $\omega_{21}$ ), and $\omega_{21} \ll \omega_{c}$ we shall have good approximation (to within one or, at least, a few percents of interaction energy) $V \simeq-\alpha E^{2} / 2$ (where $\left.\alpha=\alpha\left(\omega_{2}-\omega_{1}=\omega_{21}\right)\right)$ instead of Eq. (3). Thus the nonlinear dipole moment related to SRSS is (see, for example, [27]) $d=\partial V / \partial E=\alpha \cdot E$, and nonlinear contribution to the polarization of the medium included in Eq. (2) is $P_{n l}=N \cdot\langle\alpha\rangle \cdot E$, where an average value of a polarizability $\langle\alpha\rangle=S p(\alpha \rho)$ can be derived by using the density matrix $\rho$. Values of polarizabilities of singly ionized rare gases (in ground state) heavier than $\mathrm{He}$ are less than approximately by a factor of 2 as compared to their neutrals. For example, $\mathrm{Ne}^{+}$and $\mathrm{Ne}$ have the values of the static polarizabilities $0.21 \times 10^{-24} \mathrm{~cm}^{3}$ and $0.395 \times 10^{-24} \mathrm{~cm}^{3}$, respectively [28]. So with a knowledge of the dynamic polarizability for $\mathrm{Ar}$ at $800 \mathrm{~nm}$, that equal to about $1.64 \times 10^{-24} \mathrm{~cm}^{3}$ [28,29], and taking into account above-mentioned increasing its at two-photon resonance the value of the matrix element of the polarizability $\left|\alpha_{12}\right|$ (corresponding to the transition ${ }^{2} \mathrm{P}_{3 / 2}-{ }^{2} \mathrm{P}_{1 / 2}$ in $\mathrm{Ar}^{+}$) can be estimated to be about $10^{-21} \mathrm{~cm}^{3}$. As regards $\mathrm{Kr}$, the value of its static polarizability $2.48 \times 10^{-24} \mathrm{~cm}^{3}$ [28] is larger but comparable to Ar value. However, the 
fine structure ${ }^{2} \mathrm{P}_{3 / 2}^{0}-{ }^{2} \mathrm{P}_{1 / 2}^{0}$ levels of $\mathrm{Kr}^{+}$ions (where ${ }^{2} \mathrm{P}_{3 / 2}^{0}$ is ground state) forms a two-level system with the frequency transition $\omega_{21} / \pi c=5370 \mathrm{~cm}^{-1}$, and all other levels are removed on $\Delta>112828 \mathrm{~cm}^{-1}$ [23]. Thus to satisfy the condition SRSS (1) for $\mathrm{Kr}^{+}$it is necessary to have a laser pulse with duration $\tau \leq 6$ fs. On the other hand, the dynamical Stark shift

$\Delta \omega_{s t}=\left(\alpha_{11}-\alpha_{22}\right) \frac{E^{2}}{2 \hbar}$

$\left(\alpha_{11}, \alpha_{22}\right.$ are values of the diagonal matrix elements of the polarizability) of the resonant levels $\mathrm{Kr} 6 \mathrm{p}[5 / 2]_{2}$ and the nearest upper $6 \mathrm{p}[3 / 2]_{1}$ and lower $6 \mathrm{p}[1 / 2]_{1}$ (about $200 \mathrm{~cm}^{-1}$ ) was estimated as $\Delta \omega_{s t} \leq 40 \mathrm{~cm}^{-1}$ for intensity $I=1 \mathrm{TW}$ by Kittelmann et al. in their work [25] on direct observation of two-photon coherent response in $\mathrm{Kr}$ by femtosecond UV-laser pulses. This permits in our case to neglect the dynamical Stark shift that connected to the diagonal matrix elements of the polarizability in $\mathrm{Ar}^{+}$ (for the $3 \mathrm{p}^{5}-{ }^{2} \mathrm{P}_{3 / 2}^{0}$ and $3 \mathrm{p}\left({ }^{2} \mathrm{P}_{1 / 2}^{0}\right)$ states ) for intensities $I \leq 10 \mathrm{TW}\left(\Delta \omega_{s t}<\omega_{21}\right)$.

The average value of a polarizability $\langle\alpha\rangle$ and difference of the level populations $w=\rho_{22}-\rho_{11}$ ( $\rho$ is the density matrix) are described by the equations $[21,30]$ :

$\frac{\partial^{2}\left\langle\alpha^{\prime}\right\rangle}{\partial t^{2}}+\frac{2}{T_{2}} \cdot \frac{\partial\left\langle\alpha^{\prime}\right\rangle}{\partial t}+\omega_{21}^{2}\left\langle\alpha^{\prime}\right\rangle=-\omega_{21} \Omega^{(2)} \cdot w$,

$\frac{\partial w}{\partial t}+\frac{\left(w-w_{0}\right)}{T_{1}}=\frac{\Omega^{(2)}}{\omega_{21}}\left(\frac{\partial\left\langle\alpha^{\prime}\right\rangle}{\partial t}+\frac{\left\langle\alpha^{\prime}\right\rangle}{T_{2}}\right)$,

where

$\left\langle\alpha^{\prime}\right\rangle=\frac{\langle\alpha\rangle}{\left|\alpha_{12}\right|}$,

$\left|\alpha_{12}\right|$ is a value of the matrix element of the polarizability corresponding to the Raman transition with frequency $\omega_{21}$,

$\Omega^{(2)}=\left|\alpha_{12}\right| \frac{E^{2}}{\hbar}$

is the two-photon Rabi frequency. The parameter $T_{2}$ is the polarization relaxation time, and $T_{1}$ is the time of population relaxation.

The nonlinear contribution to the polarization of the medium included in Eq. (2) is given as

$P_{n l}=N \cdot\langle\alpha\rangle \cdot E$,

where $N$ is the number density of ions. For further consideration, it is convenient to introduce an angle of rotation of material variable [21]:

$\varphi(t, z)=\frac{\left|\alpha_{12}\right|}{\hbar} \cdot \int_{-\infty}^{t} E^{2}\left(t^{\prime}, z\right) d t^{\prime}$.

The quantity $S(z)=\varphi(+\infty, z)$ is the integral over the entire pulse of the two-photon Rabi frequency of the stimulated Raman process.
Taking into account that under condition two-photon resonance a polarizability is increased by three order of magnitude and even more [26] we take $\left|\alpha_{12}\right|=10^{-21} \mathrm{~cm}^{3}$ and $\left|\alpha_{12}\right|=10^{-20} \mathrm{~cm}^{3}$ for our simulation.

The numerical solutions of Eqs. (2)-(4) in the case of a coherent interaction for a laser pulse with $S(0)=2 \pi$ and for various $\beta \cdot z$, where $\beta=2 \pi N\left|w_{0}\right| \cdot\left|\alpha_{12}\right| \omega_{21} / c$, were carried out in previous our works [17-21]. [The quantity $\beta^{-1}$, which has the dimension of length, can be interpreted as the length of coherent SRSS.] It is convenient for the further investigation to define a dimensionless constant $\gamma=2 \pi N\left|\alpha_{12}\right|$, then $\beta=\gamma\left|w_{0}\right| \omega_{21} / c$.

The results for the $2 \pi, 4 \pi$, and $6 \pi$-pulses (16-fs and shorter) at $\gamma=0.2$ and $\beta \cdot z \leq 2$ shows that when the medium is initially unexited $\left(w(0, z)=w_{0}=\right.$ $-1,\langle\alpha(0, z)\rangle=0$ ), no more than one per cent (within $1 \%$ accuracy of the simulation) of the pulses energy could be scattered in the reverse direction. It is therefore a very good approximation to introduce a reduced wave equation [13-16]:

$\frac{\partial E}{\partial z}+\frac{1}{v} \frac{\partial E}{\partial t}=-\frac{4 \pi v}{c^{2}} \frac{\partial P_{n l}}{\partial t}$,

which describes only the forward scattering of the field. When $\delta \ll 1$, the laser pulse energy is altered by the law $[17,19]$

$\frac{d S}{d z}=\beta(1-\cos S)$

which is practically the same as for the case of a coherent direct two-photon interaction of a field with a resonant medium [5]. The Eq. (9) is the "two-photon area theorem" derived for the first time by Belenov and Poluektov [5] for direct two-photon resonant transitions. A laser pulse with $S(0)=2 \pi$ can propagate through medium $(\beta \cdot z \approx 1)$ in the SIT at SRSS regime $[17,19]$. Propagation of the pulses with $S=2 \pi$ leads to effective self-broadening of the spectrum and the generation of a spectral supercontinuum due to the SRSS [17-21].

\section{Results of numerical simulation}

We investigated the dynamics of a laser pulse having initially a Gaussian shape given by

$E(t, 0)=E_{0} \sin \left(\omega_{0} t\right) \exp \left\{-2 \ln 2 \cdot\left(\frac{t}{\tau_{0}}\right)^{2}\right\}$,

where $E_{0}$ is the initial peak field amplitude and $\omega_{0}$ is the initial frequency of the field that propagates through the medium, initially unexited (i.e., $w(0, z)=w_{0}=$ $-1,\langle\alpha(0, z)\rangle=0)$. We used foregoing parameters for $\mathrm{Ar}^{+}, \gamma=2 \pi N\left|\alpha_{12}\right| \simeq 0.2$ (for example, $N=3 \times$ $10^{18} \mathrm{~cm}^{-3}$ and $\left|\alpha_{12}\right|=10^{-20} \mathrm{~cm}^{3}$ or $N=3 \times 10^{19} \mathrm{~cm}^{-3}$ and $\left|\alpha_{12}\right|=10^{-21} \mathrm{~cm}^{3}$ ), then $\beta \simeq 1.8 \times 10^{3} \mathrm{~cm}^{-1}$, and $S(0)=2 \pi$ at $\mathrm{I} \simeq 10^{12} \mathrm{~W} / \mathrm{cm}^{2}$. 


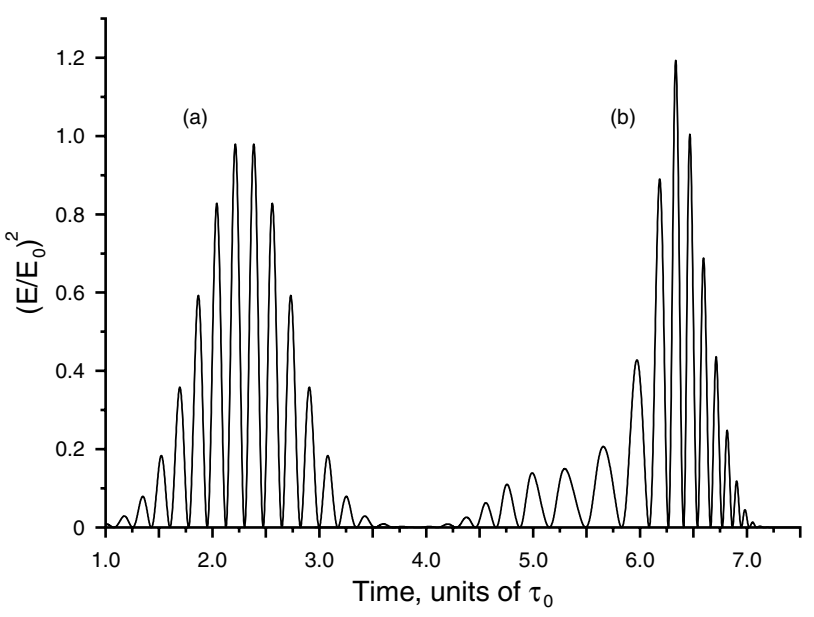

Figure 1 Dynamics of squared field E (normalized to initial amplitude $\mathrm{E}_{0}$ ) vs. time (normalized to initial pulse width $\tau_{0}$ ) for $\tau_{0}=8$ fs, $S(0)=2 \pi, \gamma=0.2$. (a) $\beta z=0$; (b) 1.4

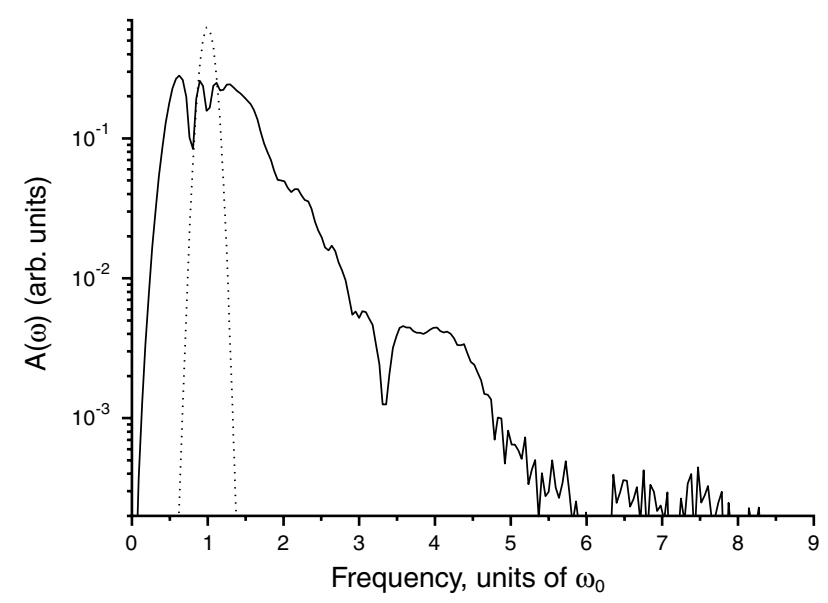

Figure 2 Amplitude spectra $\mathrm{A}(\omega)$ of field E, corresponding to Fig. 1. The dotted line is spectrum of initial field (Fig. 1a $\beta z=$ $0)$. The firm line is spectrum of field after distance corresponding to $\beta z=1.4$ (Fig. 1b)

The dynamics of the field and spectrum of the 8-fs pulse with $S=2 \pi$ and three optical cycle within the FWHM of intensity (at $800 \mathrm{~nm}$ ) is shown in Figs. 1 and 2. The energy of the $2 \pi$-pulses that propagating in conditions of SIT at SRSS is conserved (see Eq. (9) and [19]). But in contrast to one-photon SIT [1-3], and direct two-photon SIT [5], the $2 \pi$-pulses under SRSS are nonstationary both in shape and in spectrum (see Figs. 1,2). The field on the leading edge of the pulse transfers the medium to the excited state, and the amplitude and frequency of the its leading edge are decreased. The wavelength of this portion of the field is shifted toward the red (continuous Stokes shifting)(see Fig. 1b). The field of the following portion of the

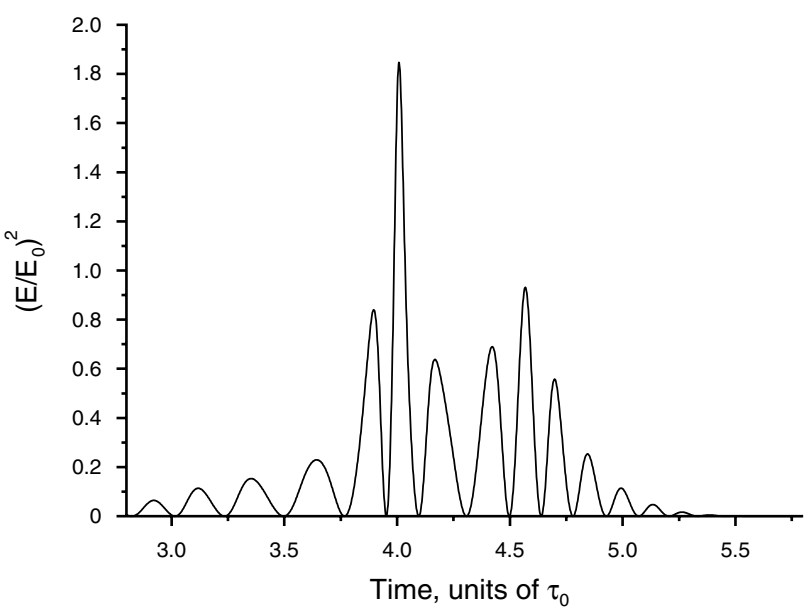

Figure 3 Dynamics of squared field E (normalized to initial amplitude $\mathrm{E}_{0}$ ) vs. time (normalized to initial pulse width $\tau_{0}$ ) for $\tau_{0}=8$ fs, $S(0)=4 \pi, \gamma=0.2$, and $\beta z=0.68$

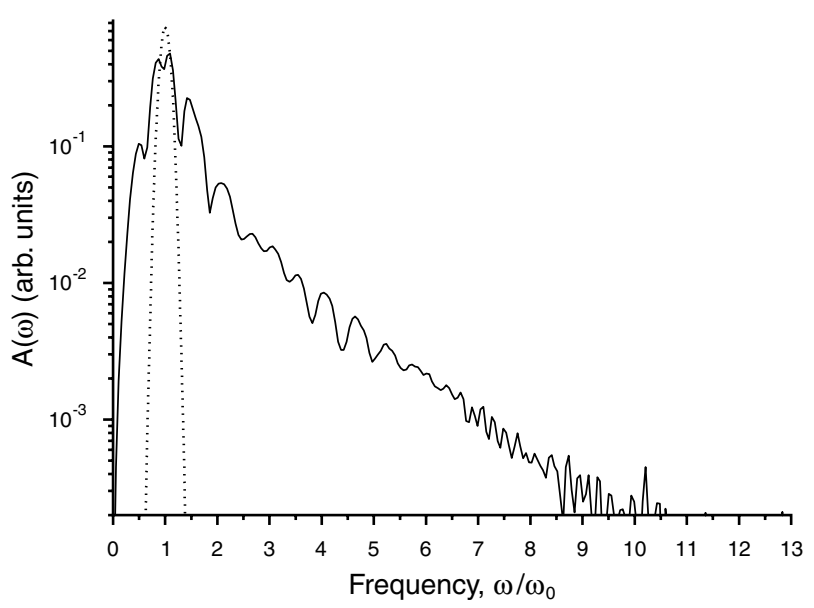

Figure 4 Amplitude spectra $\mathrm{A}(\omega)$ of field $\mathrm{E}$, corresponding to Fig. 3. The dotted line is spectrum of initial field $(\beta z=0)$. The firm line is spectrum of field after distance corresponding to $\beta z=0.68$

laser pulse causes a coherent transfer of the medium to the initial state, resulting in an increase in amplitude to the trailing part of the pulse. The wavelength of this portion of the field is shifted toward the blue (continuous anti- Stokes shifting)(see Fig. 1b). So the pulse, during propagating, generates a spectral supercontinuum (Fig. 2). During this process, the overall energy of the laser pulse is conserved, but a large share of the energy is moved into the higherfrequency band.

The dynamics of the field and spectrum of $4 \pi$-pulses ( $S=4 \pi)\left(\tau_{0}=8\right.$ fs FWHM, $\left.800 \mathrm{~nm}\right)$ is shown in Figs. 3 and 4 , respectively. The splitting (or pulse breakup) of the $4 \pi$-pulse into two pulses is characteristic of all types 


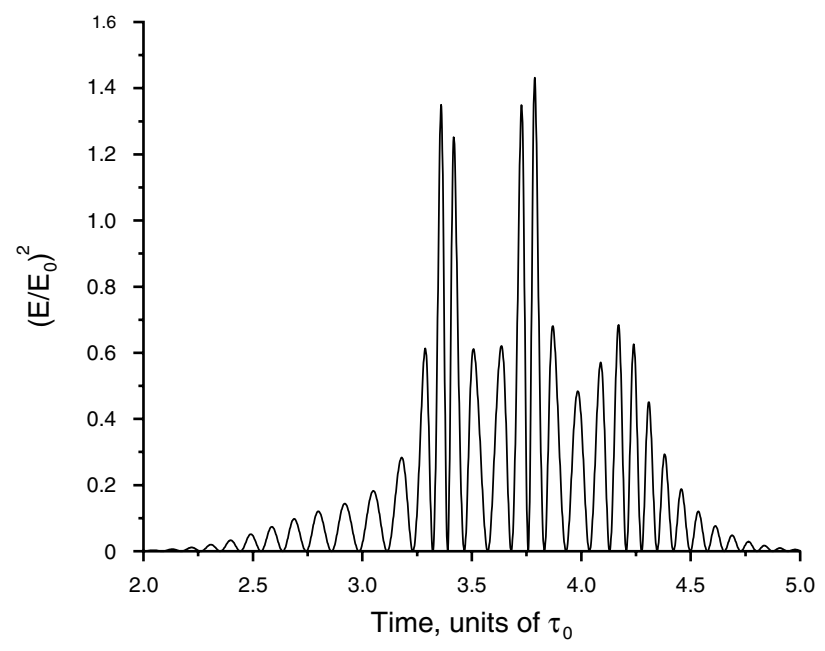

Figure 5 Dynamics of squared field E (normalized to initial amplitude $\mathrm{E}_{0}$ ) vs. time (normalized to initial pulse width $\tau_{0}$ ) for $\tau_{0}=16$ fs, $S(0)=6 \pi, \gamma=0.2$, and $\beta z=0.68$

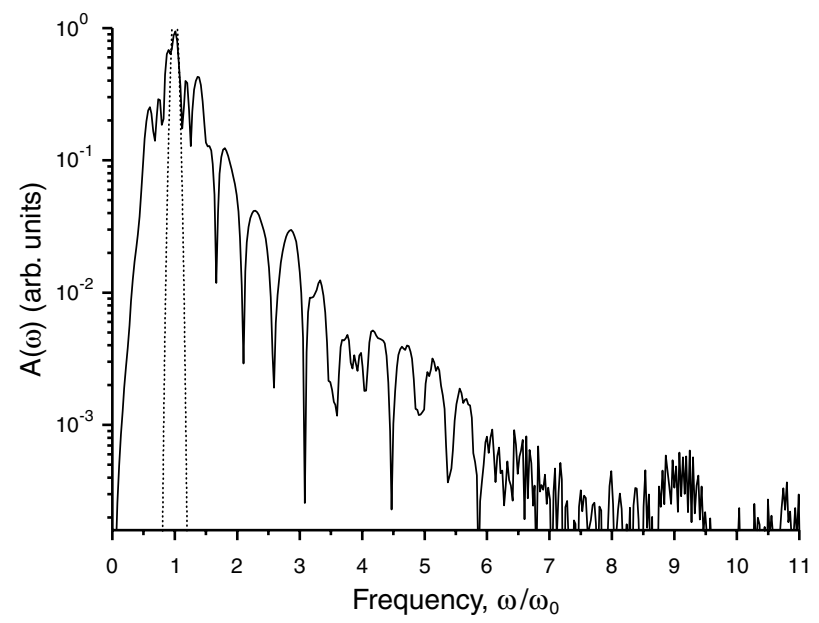

Figure 6 Amplitude spectra $A(\omega)$ of field $E$, corresponding to Fig. 5. The dotted line is spectrum of initial field $(\beta z=0)$. The firm line is spectrum of field after distance corresponding to $\beta z=0.68$

of SIT, and is also evident here for the SIT at SRSS. In this case, the forward pulse is compressed into a half-cycle electromagnetic field (FWHM).

The dynamics of the field and spectrum of $6 \pi$-pulses $(S=6 \pi)\left(\tau_{0}=16 \mathrm{fs}\right.$ FWHM, $\left.800 \mathrm{~nm}\right)$ is shown in Figs. 5 and 6, respectively. The splitting (or pulse breakup) of the $6 \pi$-pulse into three $2 \pi$-pulses also can see in Fig. 5 . However, in this case first and second $2 \pi$-pulses are compressed into one-cycle electromagnetic fields of near amplitude values.

It is necessary to note that we have the exact solutions of the full system of the Eqs. (4)-(6), and (8), without the strong requirement $\delta \ll 1$. This requirement, however, was used to derive Eq. (9) from Eqs. (4)-(6), and (8) by neglecting the term $\omega_{21}^{2}\langle\alpha\rangle$ in Eq. (4) [16]. For the numerical simulations we used an improved version of the code presented in [19-21].

Finally, we have calculated the effect of the plasma on coherent lengths. The spectra of pulses (Figs. 2,4,6) do not have frequencies that exceeds $\omega=11 \omega_{0}$ (at last, less then $10^{-3}$ on amplitude level). Thus it is enough, in our case, to calculate a coherent length $L_{c}$ of 11-th harmonic. If densities of ions and free electrons are $3 \times 10^{18} \mathrm{~cm}^{-3}$, then the electron plasma frequency is $\omega_{p}=8 \times 10^{13} \mathrm{~s}^{-1}$, and the coherent length $L_{c}=\pi /\left(11 k_{1}-k_{11}\right) \simeq 42.4 \mu \mathrm{m}$, where the wave number of the $\mathrm{j}$-th harmonic is given by

$k_{j}=\frac{\omega_{j}}{c} \sqrt{1-\left(\frac{\omega_{p}}{\omega_{j}}\right)^{2}}, \quad j=1,11$,

$\omega_{1}=\omega_{0}, \omega_{11}$ is the frequency that equal 11-th harmonic. It is easy to see that, for the parameters used above, the propagation distances $z \simeq 8 \mu \mathrm{m}$ (Fig. 1) and $z \simeq 4 \mu \mathrm{m}$ (Figs. 3 and 5) are much less then this coherent length $L_{c} \simeq 42.4 \mu \mathrm{m}$.

\section{Summary}

We have shown that two-photon Raman-type self-induced transparency for few-cycle laser pulses have new properties as compared with one-photon SIT: (1) nonstationary fields not only in $6 \pi$-pulses, $4 \pi$-pulses but in $2 \pi$ pulses; (2) the possibility of self-compression and selfamplification of laser pulses containing several oscillation periods into pulses containing one- or half-cycle fields (FWHM) with higher frequencies and amplitudes. The simulations show that in the regime of SIT at the SRSS the nonlinear transformation of $2 \pi$-pulses causes an increase in the peak intensity of the later portion of the laser pulse, while conserving the overall energy in the pulse. A continuous spectral shifting of the increasing portion of the field towards higher frequencies accompanies the effect. The leading edge pumps the Raman quantum transition coherently, decreasing its intensity and shifting its spectrum towards lower frequencies. Such energy repumping permits to generate single as well as double attosecond pulses with very high efficiency [31].

Acknowledgements This work was partially supported by the B.I.Stepanov Institute of Physics NASB, "Coherence" Program (grant "Coherence-62"), by the BSU, and by the ISTC Project B-1065.

\section{References}

[1] S.L. McCall and E.L. Hahn, Phys. Rev. Lett. 18, 908 (1967); S.L. McCall and E.L. Hahn, Phys. Rev. 183, 457 (1969).

[2] R.E. Slusher and H.M. Gibbs, Phys. Rev. A 5, 1634 (1972); R.E. Slusher and H.M. Gibbs, Phys. Rev. A 6, 1255 (1972). 
[3] L. Allen and J.H. Eberly, Optical Resonance and Two-level Atoms (Dover, New York, 1987).

[4] Y.R. Shen, The Principles of Nonlinear Optics (Wiley, New York, 1984)

[5] E.M. Belenov and I.A. Poluektov, Zh. Eksp. Teor. Fiz. 56, 1407 (1969); E.M. Belenov and I.A. Poluektov, J. Exp. Theor. Phys. 29, 754 (1969).

[6] N. Tan-no, K. Yokoto, and H. Inaba, Phys. Rev. Lett. 29, 1211 (1972).

[7] V.A. Gridin, et al., Pisma Zh. Eksp. Teor. Fiz. 28, 440 (1978).

[8] I.A. Poluektov, Yu.M. Popov, and V.S. Roitberg, Pisma Zh. Eksp. Teor. Fiz. 18, 638 (1973).

[9] I.A. Poluektov, Yu.M. Popov, and V.S. Roitberg, Pisma Zh. Eksp. Teor. Fiz. 20, 533 (1974).

[10] R.T. Hodgson, P.P. Sorokin, and J.J. Wynne, Phys. Rev. Lett. 32, 343 (1974); R.L. Carman, Phys. Rev. A 12, 1048 (1975); D. Grischkowsky, M.M.T. Loy, and P.F. Liao, Phys. Rev. A 12, 2514 (1975); J.-C. Diels and A.T. Georges, Phys. Rev. A 19, 1589 (1979).

[11] N. Tan-no, K. Yokoto, and H. Inaba, J. Phys. B 8, 339, (1975); N. Tan-no, K. Yokoto, and H. Inaba, J. Phys. B 8, 349, (1975).

[12] T. Brabec and F. Krausz, Rev. Mod. Phys. 72, 545 (2000).

[13] R.K. Bullough and F. Ahmad, Phys. Rev. Lett. 27, 330 (1971); J.C. Eilbeck, J.D. Gibbon, P.J. Caudrey, and R.K.Bullough, J. Phys. A 6, 1337 (1973).

[14] P.J. Caudrey, J.D. Gibbon, and J.C. Eilbeck, Phys. Rev. Lett. 30, 237 (1973).

[15] R.K. Dodd, J.C. Eilbeck, J.D. Gibbon, and H.C. Morris, Solitons and Nonlinear Wave Equations (Academic Press, London, 1982).

[16] E.M. Belenov, A.V. Nazarkin, and I.P. Prokopovich, JETP Lett. 55, 218 (1992).
[17] E.M. Belenov and I.P. Prokopovich, Quantum Electron. 23, 497 (1993).

[18] E.M. Belenov, P.G. Kryukov, A.V. Nazarkin, and I.P. Prokopovich, J. Exp. Theor. Phys. 78, 15 (1994).

[19] E.M. Belenov and I.P. Prokopovich, J. Russ. Laser Res. 15, 283 (1994).

[20] I.P. Prokopovich and A.A. Khrushchinskii, Laser Phys. 7, 305 (1997).

[21] I.P. Prokopovich and J. Peatross, Laser Phys. 9, 588 (1999); I.P. Prokopovich and J. Peatross, Proc. SPIE 4752, 31 (2002).

[22] S.A. Akhmanov, V.A. Vyslouch, and A.S. Chirkin, Optics of Femtosecond Laser Pulses (AIP, New York, 1992).

[23] A.A. Radzig and B.M. Smirnov, Parameters of atoms and atoms ions (Energoatomizdat, Moscow, 1989), in Russian.

[24] R.W. Boyd, Nonlinear Optics (Academic Press, London, 1992).

[25] O. Kittelmann, J. Ringling, A. Nazarkin, et al., Phys. Rev. Lett. 76, 2682 (1996).

[26] J.F. Reintjes, Nonlinear Optical Parametric Processes in Liquids and Gases (Academic Press, London, 1984).

[27] L.D. Landau and E.M. Lifshits, Quantum Mechanics (Pergamon, Oxford, 1977).

[28] E.W. McDaniel and E.A. Mason, The mobility and diffusion of ions in gases (Wiley, New York, 1973).

[29] P.J. Leonard, At. Data Nucl. Data Tables 14, 21 (1974).

[30] R.H. Pantell and H.E. Puthoff, Fundamentals of Quantum Electronics (Wiley, New York, 1969).

[31] I.P. Prokopovich, Single and double attosecond pulses: resonance generation and propagation in $\mathrm{Ar}^{+}, 13$ th International Laser Physics Workshop, (Trieste, July 12-16, 2004); Laser Phys. (to be published). 